\title{
Computational simulation of an altitude adaptive nozzle concept
}

\author{
K. Schomberg ${ }^{1, a}$, G. Doig ${ }^{1, b}$ and J. Olsen ${ }^{1, c}$ \\ ${ }^{1}$ School of Mechanical and Manufacturing Engineering, The University of New South Wales, \\ Kensington, NSW 2052, Australia
}

akyll.schomberg@unsw.edu.au, bg.doig@unsw.edu.au, cj.olsen@unsw.edu.au

Keywords: propulsion, expansion-deflection, nozzle, altitude adaption, CFD

\begin{abstract}
A computational analysis of an annular converging-diverging (CD) and an altitude adaptive expansion-deflection (ED) nozzle is presented. Numerical results were generated using a 2D axisymmetric, pressure-coupled solver in conjunction with the Spalart-Allmaras turbulence closure model and second order spatial discretisation schemes. Results were recorded over a theoretical altitude range and compared to experimental static pressure readings and schlieren images. The correlation between numerical and experimental static pressure values was high for all cases. Comparison of schlieren imagery outlined the large variety of flow regions within the ED nozzle flow field. The interactions between these regions were highly sensitive to turbulence and reinforced that conventional inviscid analytical techniques are unable to accurately describe behaviour within the ED nozzle flow field. The results highlight the salient effect of viscous effects within the ED nozzle flow field and justify a continued approach utilising computational fluid dynamics to increase understanding of the ED nozzle concept.
\end{abstract}

\section{Introduction}

The increasing cost of transporting payload into orbit has in turn increased demand for a highly efficient, reusable, single stage launch system. Conventional propulsion systems operating from sea to level to vacuum currently suffer from an altitude-induced performance loss of up to $15 \%$ [1]. This performance loss is a result of conventional fixed-area nozzles producing constant exhaust properties at the nozzle exit. Optimal nozzle efficiency exists when the conditions of the exhaust exiting the nozzle are equal to that of the receiver. However, as the receiver or atmospheric conditions vary with altitude, optimal efficiency can only occur at one specific altitude and losses are incurred at all other points within the flight path. Altitude adaptive nozzle concepts that overcome these inefficiencies have existed for over 50 years [2]. These concepts fall into two general classes: passive area variation nozzles [3, 4] and controlled flow separation devices [5]. Controlling the conditions for flow separation is difficult [6], and can result in damaging side loads to the nozzle walls [7]. Additionally, these devices compensate for altitude through an inherent staging process and are therefore unable to truly minimise altitude losses [8].

Variable area nozzle concepts employ a passive and continual variation of the effective nozzle exit area to adapt for the change in receiver pressure to and ensure efficiency remains high throughout the entire flight path. This variation of nozzle exit area is achieved through an interaction between the supersonic exhaust and the receiver, usually ambient air. This interaction occurs externally in the case of the plug nozzle [3] or internally in the case of the expansiondeflection (ED) [4] nozzle. Recent work has shown comparable potential for both concepts [9], with the increased heat flux and transonic region instability of the plug nozzle [10] prompting further investigation into the ED concept.

Current ED nozzle design methodology relies strongly on analytical methods that assume inviscid solutions for the nozzle flowfield [9]. However, the integral viscous wake region is unable to be fully described utilising these techniques. A computational fluid dynamics (CFD) approach enables the entire flowfield to be modelled, and has been shown to be capable of handling complex flow behaviour similar to that present within the ED nozzle [11]. Considering the gain in flowfield knowledge from these studies and the availability of increased computational resources, the current analytical methods have been rendered unnecessary. Therefore, to further the 
understanding of flow behaviour within the ED nozzle, a thorough analysis utilising CFD is required. In the present work, a RANS-based computational analysis of an ED nozzle and an equivalent $\mathrm{CD}$ nozzle has been conducted to determine the relative performance between these configurations in addition to the significance of turbulence within the ED nozzle flowfield.

\section{Numerical model}

The geometry utilised in all simulations was consistent with that used in a prior experiment [12]. This consisted of a fixed nozzle shroud and interchangeable flow deflectors, herein referred to as a 'pintles' to vary configurations between the CD and the ED nozzles. Isentropic design methods were utilised for the divergence contour and the configuration manipulated to support the pintle attachments. Fig. 1 shows the nozzle geometry as well as general setup of the experimental configurations and locations of the pressure tapping points.

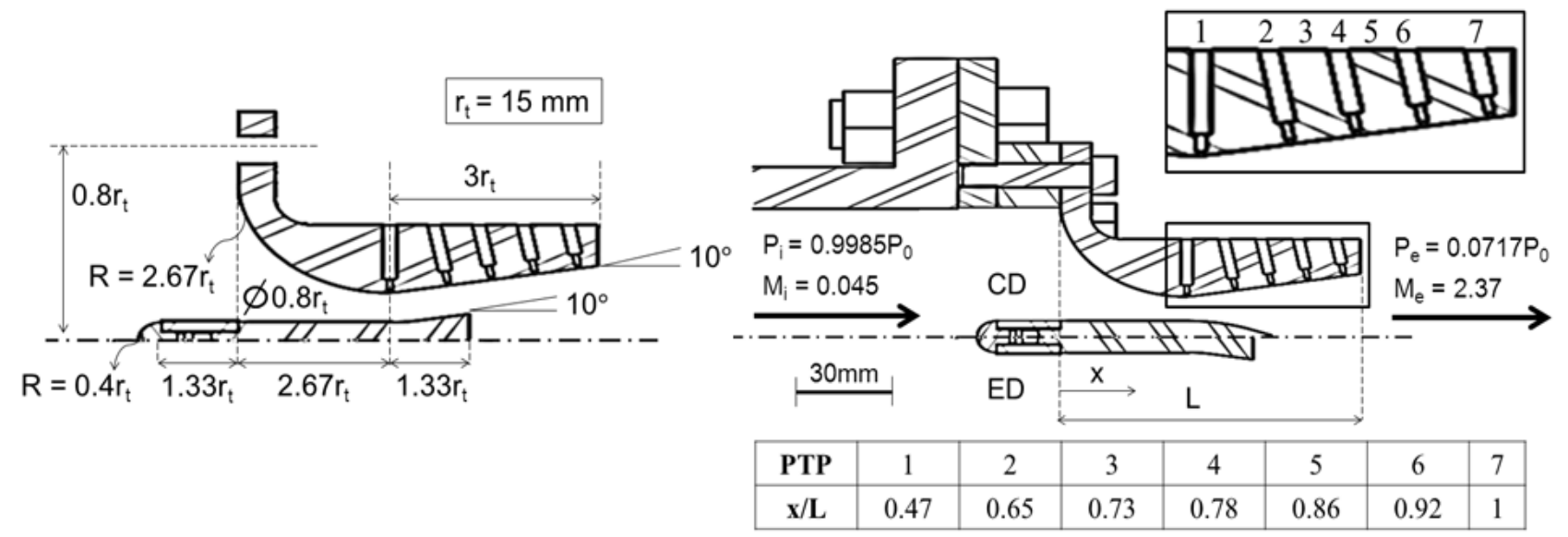

(a)

(b)

Figure 1: Sectioned image of the (a) ED nozzle geometry and (b) CD and ED nozzle configurations.

The flow through both nozzle configurations was treated as compressible and turbulent. To verify and validate the numerical model, initial solver parameters were required. The baseline turbulence model used was the Spalart-Allmaras (SA) model, which is a one-equation turbulence model developed specifically for aerodynamic flowfields involving wall bounded flows [13]. Turbulent intensity and length scale were used to evaluate turbulent properties. Initial values for these parameters were calculated from the Reynolds number and boundary layer thickness at the nozzle inlet and resulted in a turbulent intensity of $3.6 \%$ and length scale of $1.68 \mathrm{~mm}$. The pressurebased coupled solver and second order spatial discretisation schemes were utilised for all simulations. To assess numerical convergence, surface monitors calculating mass flow rate were set at the nozzle inlet, outlet, throat and exit plane. The solution was deemed to have converged when the mass flow rate at all surface monitors changed by less than $0.01 \%$ over 500 iterations in conjunction with a mass flux of less than $1 \%$ between the inlet and outlet mass flow rates within the domain.

A fully structured mesh comprised of quadrilateral cells was used for all models. To enable accurate resolution of the boundary layer, prism inflation layers with a viscous length $\left(\mathrm{y}^{+}\right)$of 1 at the nozzle wall were used to maintain the presence of cells within the viscous sub-layer. Grid convergence was assessed in the CD configurations across a coarse, standard and fine level of mesh comprised of $1.25,2$ and $3.38 \times 10^{5}$ cells respectively. Mesh refinement had a minimal effect on pressure distribution as shown in Fig. 2. To strengthen the verification process, turbulent intensity and length scale were both increased and decreased by an order of magnitude. This variation had a negligible effect on pressure distribution and the location of the shock waves, whereas the resolution of the shock itself was shown to be sensitive to these parameters. However, the periodic adaption of the mesh with respect to normalised density gradient annulled these effects and was used for all models. 
To assess effects on the description of turbulence within the flowfield, the SA, k-omega shear

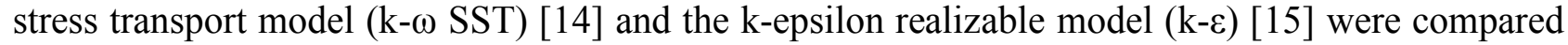
to an invsicid model. The inviscid model failed to predict flow separation, whereas the k- $\omega$ SST model prematurely predicted separation as shown in Fig. 2. The SA and k- $\varepsilon$ models had a similar pressure distribution; however the dissipative nature of the $\mathrm{k}-\varepsilon$ model negatively affected the comparison of schlieren images. The standard mesh and SA turbulence model were selected to generate all numerical results due to a close correlation with pressure readings and improved accuracy of schlieren comparison.

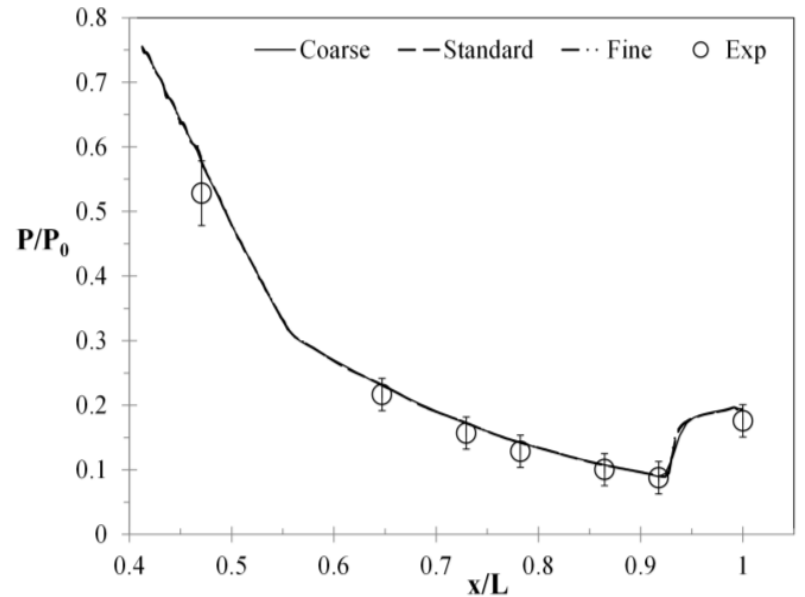

(a)

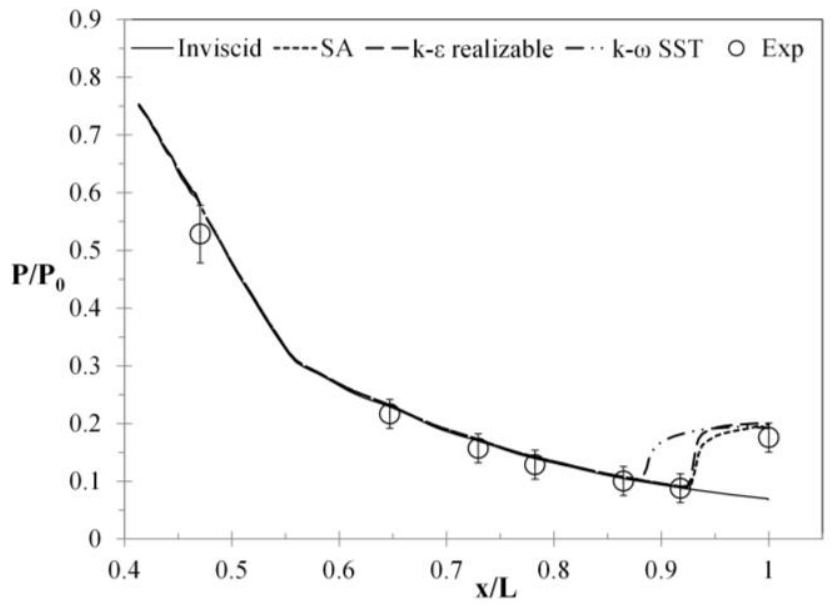

(b)

Figure 2: Effect of (a) mesh level and (b) turbulence model on pressure distribution in the CD configuration.

\section{Results}

To evaluate flow behaviour over a theoretical altitude range, operating pressure ratios of 4.5, 5.4 and 6.7 were used. Results were obtained through the evaluation of thrust parameters as well as the comparison of numerical and experimental schlieren images and pressure distributions throughout the divergent section of both nozzle configurations. Thrust parameters were calculated from weighted-average values at the nozzle exit plane. Theoretical and ideal pressure curves were generated using isentropic flow relations for the physical and ideal geometries respectively. All experimental results were taken from previous work [12].

Low pressure inlet. The 4.5 bar inlet represented the minimum pressure ratio of all the conditions tested. This operating condition exceeded the typical overexpansion limits allowed in conventional designs; as the onset of flow separation would be expected to occur. Figure 3 compares the pressure distribution and schlieren images in the CD and ED configuration and Table 1 details thrust parameters for both models.

A high correlation between experimental and numerical pressure values was evident in both configurations. Static wall pressures were greater in the ED nozzle due to the effect of compression waves forming downstream of the pintle. The earlier location of flow separation in the CD configuration was reinforced by the higher exit pressure and corresponding lower exit velocity as a result of the pressure recovery effect. Consistency between the schlieren images was less pronounced for the ED configuration. The numerical model captured the supersonic jet region of the flow relatively well but appeared to break down across the wake region. The result may represent a potential limitation of the SA model in capturing propagation of the free shear layer and subsequent shock reflection between the interacting flow fields, in spite of the initial flow structure being evident. The greater specific impulse $\left(\mathrm{I}_{\mathrm{SP}}\right)$ and thrust value calculated for the ED configuration was expected due to the location of separation. This result reinforced the positive effect of the wake region in delaying the onset of flow separation and increasing thrust values. 
Table 1: Thrust parameters for the low pressure inlet condition

\begin{tabular}{lllll}
\hline \hline Model & Exit velocity, $\mathrm{ms}^{-1}$ & Exit pressure, $\mathrm{Pa}$ & Thrust, $\mathrm{N}$ & ISP \\
\hline \hline CD & 393.83 & 73513 & 308.24 & 34.564 \\
ED & 468.08 & 56359 & 347.11 & 38.717 \\
\hline
\end{tabular}
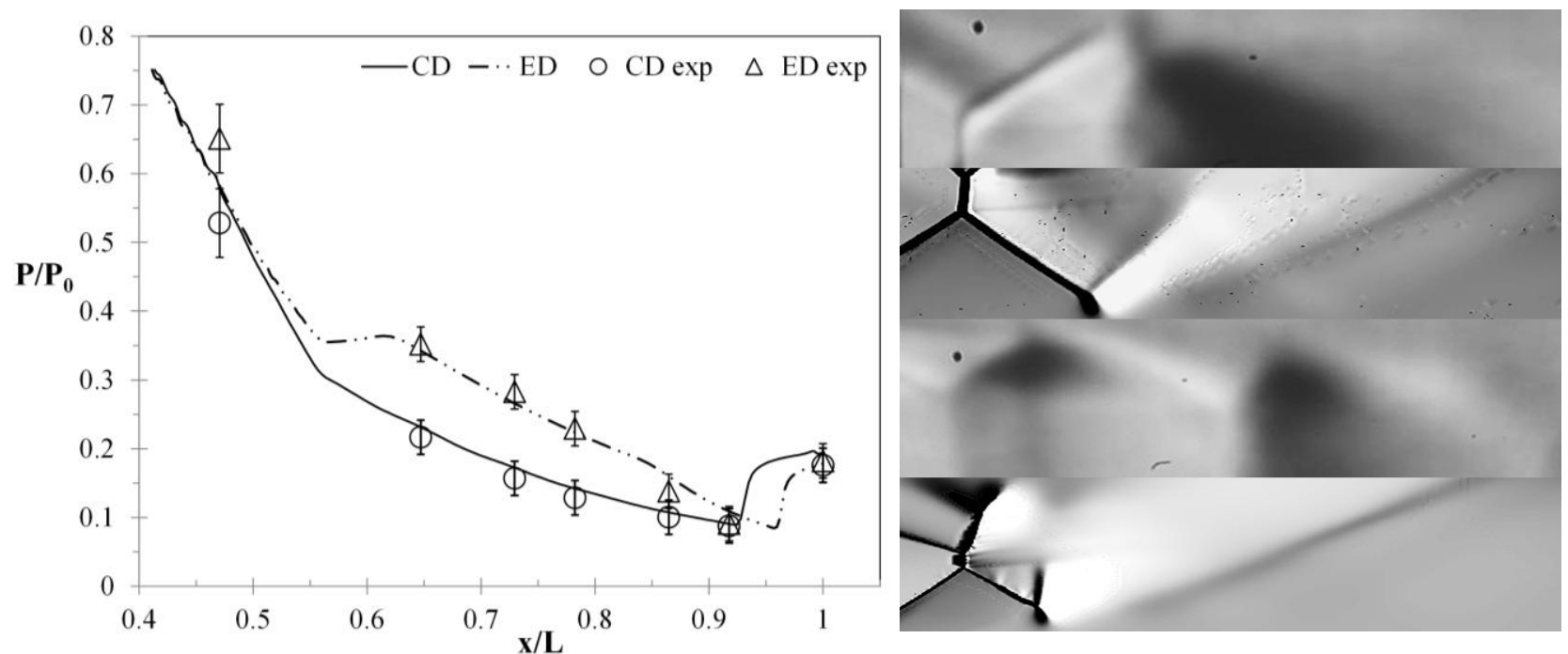

Figure 3: Low pressure distributions and schlieren comparison in the CD (top) and ED (bottom) configurations.

Baseline pressure inlet. The 5.4 bar inlet was taken as the baseline condition for results. This flow condition represented the theoretical limit at which separation was expected to occur. Figure 4 compares the pressure distribution and schlieren images in the CD and ED configuration and Table 2 details thrust parameters for both models.

Table 2: Thrust parameters for the baseline pressure inlet condition

\begin{tabular}{lllll}
\hline \hline Model & Exit velocity, $\mathrm{ms}^{-1}$ & Exit pressure, $\mathrm{Pa}$ & Thrust, $\mathrm{N}$ & $\mathrm{I}$ \\
\hline \hline CD & 526.76 & 54784 & 471.76 & 45.860 \\
ED & 535.05 & 53896 & 476.26 & 46.284 \\
\hline
\end{tabular}
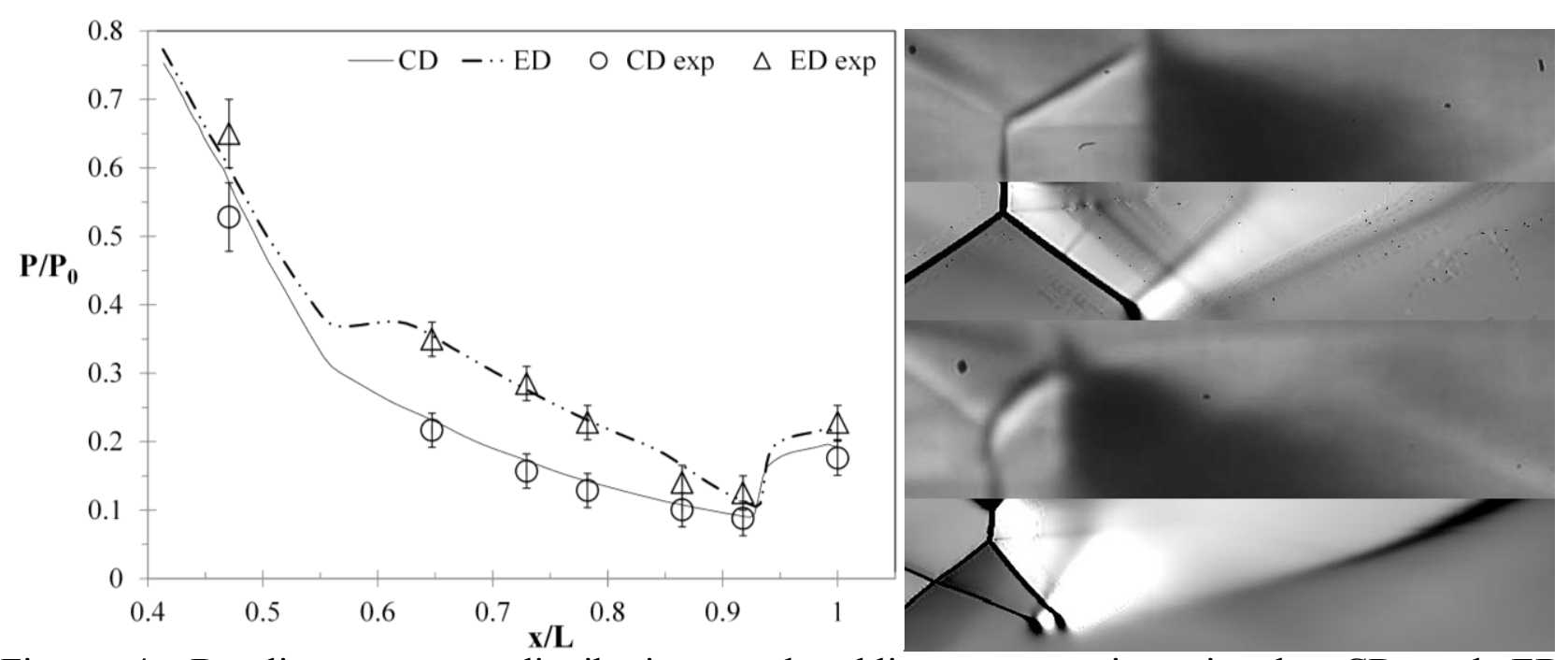

Figure 4: Baseline pressure distributions and schlieren comparison in the $\mathrm{CD}$ and ED configurations.

All numerical pressure values within the divergent section were in agreement with experimental values and the location of flow separation within $1 \%$ between both models. The correlation between schlieren images was high for both the CD and ED nozzle. The main variation in 
the ED configuration was the observable distance between the primary (separation induced) shock and the secondary (pintle induced) shock which was not evident in the experimental image. In the $\mathrm{CD}$ configuration, minor reflective shocks were present in the numerical schlieren image. The absence of a wake region in the ED nozzle appeared to be linked to the improved numerical schlieren accuracy and may limit the validity of a RANS approach to wake-dominated flow. Thrust values were much more analogous in the baseline condition which was consistent with the similar location of flow separation in addition to the presence of a shock-dominated flowfield in both configurations.

High pressure inlet. A pressure ratio of 6.7 was used to observe nozzle operation at a condition where flow separation should theoretically be avoided. Figure 5 compares the pressure distribution and schlieren images in the CD and ED configuration and Table 2 details thrust parameters for both models.

Table 3: Thrust parameters for the high pressure inlet condition

\begin{tabular}{lllll}
\hline \hline Model & Exit velocity, $\mathrm{ms}^{-1}$ & Exit pressure, $\mathrm{Pa}$ & Thrust, $\mathrm{N}$ & I \\
\hline \hline CD & 532.97 & 55444 & 559.62 & 47.437 \\
ED & 532.25 & 57907 & 561.09 & 47.754 \\
\hline
\end{tabular}

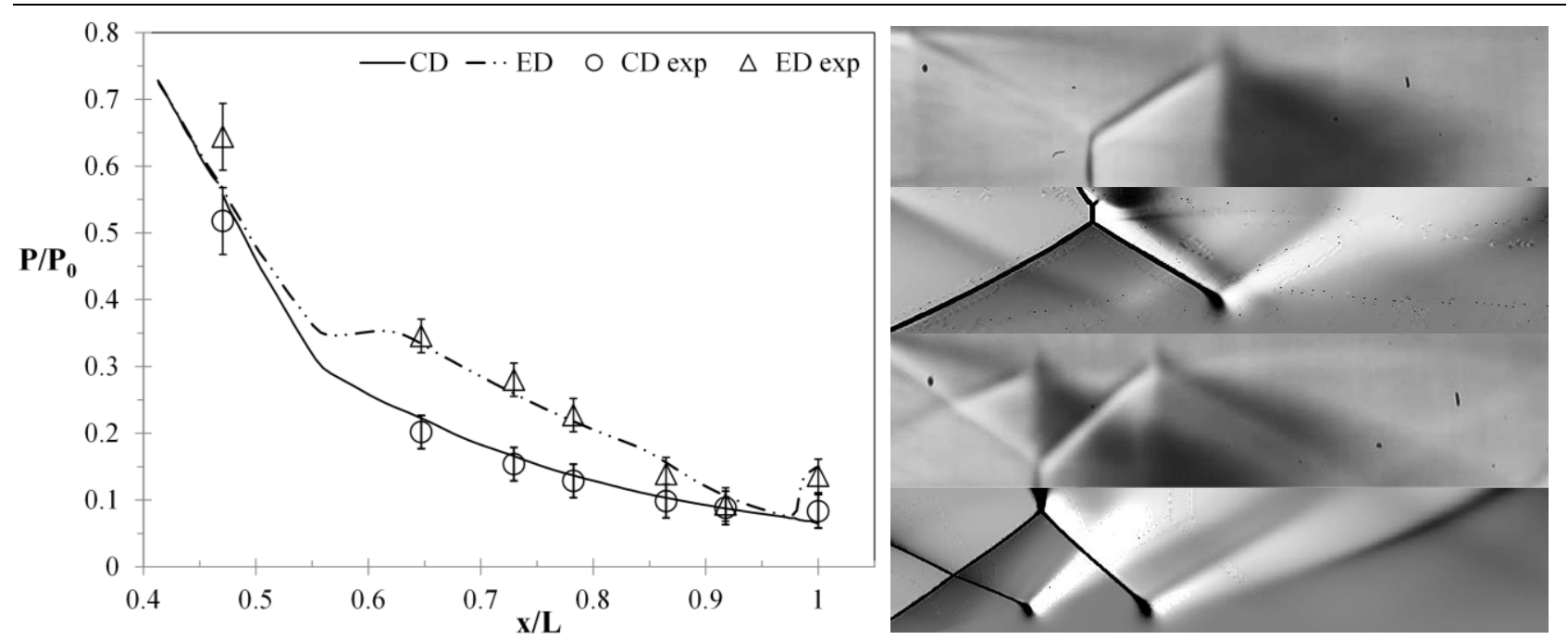

Figure 5: High pressure distributions and schlieren comparison in the CD and ED configurations.

The numerical pressure distributions were again consistent with the experimental results. Flow separation occurred in the ED nozzle and was avoided in the CD nozzle. This result was indicative of the inherent characteristic of the ED nozzle to operate independently of pressure ratio and suggests that this characteristic can be manipulated with a more comprehensive design method. Correlation between schlieren images was high for both configurations and the secondary shock present in the ED nozzle was accurately modelled. The translational location of the Mach disk in each configuration was consistent with the difference in flow separation location between models. The exit velocity was comparable between both models, with a slightly higher exit pressure in the ED nozzle resulting in a greater thrust and ISP value.

\section{Conclusion}

The correlation between numerical and experimental pressure values in the divergent section was high across all operating conditions. The discrepancy for both models was the pressure value at the throat region. This was due primarily to the large variation in pressure in the region. The location of flow separation was relatively constant in the ED configuration and suggested nozzle operation was independent of pressure ratio. This quality is believed to be inherent within the design and was reinforced by the reduced effect of separation on thrust parameters relative to the CD configuration. In the CD nozzle, the location of flow separation was consistent with theory and 
approached the nozzle exit with increasing pressure. The comparable thrust performance between configurations for the baseline and high pressure conditions was linked to the shock dominated flowfield existent in both models. The increased thrust performance in the low pressure condition outlines the benefit of the wake region in mitigating thrust losses due to overexpansion of the nozzle flow.

Comparison of Schlieren imagery suggested that the general structure of the flow was well approximated in the numerical models and reinforced the high correlation between the numerical and experimental pressure values. Discrepancies in shading and minor reflective shock details were deemed to be a result of the evaluation of numerical density gradient exclusively in the longitudinal dimension. These effects were ignored in the analysis due to the negligible effect on major flowfield detail and the evaluation of thrust parameters. The poor correlation in the low pressure ED nozzle may represent a limitation in the SA model for describing wake-dominated flowfields. Further investigation is necessary to enable accurate descriptions of ED flowfield and determine the suitability of RANS modelling under all operating conditions. The analysis confirmed the integral role of turbulence within the ED nozzle flowfield and justified the continued use of CFD over conventional inviscid analytic numerical methods.

\section{References}

[1] Sutton, G.P., Biblarz, O., Rocket Propulsion Elements. 7th ed. 2001, New York, NY: John Wiley \& Sons.

[2] Rao, G.V.R., Recent developments in rocket nozzle configurations. ARS journal, 1961: p. 1488-1494.

[3] Rao, G.V.R., Spike nozzle contour for optimum thrust. Ballistic missile and space technology, 1961. 2: p. 92-101.

[4] Rao, G.V.R., Analysis of a new concept rocket nozzle. Journal of Liquid Rockets and Propellants, 1960: p. 669-682.

[5] Nasuti, F., Onofri, M., Flow analysis and methods of design for dual bell nozzles, in 37th joint propulsion conference and exhibit 2001, AIAA: Salt lake city, UT.

[6] Ostlund, J., Supersonic flow separation with application to rocket engine nozzles, 2004, Royal Institute of Technology: Stockholm, Sweeden. p. 31.

[7] Onofri, M., Nasuti, F., The Physical Origins of Side Loads in Rocket Nozzles, in 35th Joint propulsion conference and exhibit 1999, AIAA: Los Angeles, CA.

[8] G. Hagemann, M.F., D. Manski, Critical assessment of dual bell nozzles. AIAA journal, 1997.

[9] Taylor, N.V., Sato, T., Experimental and Computational Analysis of an ED nozzle in open wake mode, in 26th Applied Aerodynamics Conference 2008, AIAA: Honolulu, HI.

[10]Hagemann, G., Immicht, H., Terhardt, M., Dumnov, G., Critical assessment of the linear plug nozzle concept, in 37th Joint propulsion conference and exhibit 2001, AIAA: Salt lake city, UT.

[11]Hagemann, G., Schwane, R., Reijasse, P., Ruf, J., Nato TRO WG 10 - CFD results of plug nozzle test cases, in 38th Joint Propulsion Conference and Exhibit 2002, AIAA: Indianpolis, IN.

[12] Schomberg, K.A., Olsen, J., Altitude compensation in expansion deflection nozzles, in $18^{\text {th }}$ Australian Fluid Mechanics Conference 2012: Launceston, Australia.

[13] Spalart, P., Allmaras, S., A one-equation turbulence model for aerodynamic flows. La Recherche Aerospatiale, 1992. 1(5).

[14]Menter, F.R., Two equation eddy-viscosity turbulence models for engineering applications. AIAA journal, 1994. 32(8): p. 1598-1605.

[15] Shih, T.H., Liou, W.W., Shabbir, A., Zhu, J., A New k-epsilon eddy-viscosity model for high Reynolds number turbulent flows - model development and validation. Computers Fluids, 1995. 24(3): p. 227-238. 